\title{
The Prognosis of Single Hormone Receptor-Positive Breast Cancer Stratified by HER2 Status
}

\author{
Hengqiang Zhao* and Yiping Gong \\ Department of Breast and Thyroid Surgery, Renmin Hospital of Wuhan University, Wuhan, China
}

OPEN ACCESS

Edited by:

Mariela Vasileva-Slaveva, University Hospital Acibadem City

Clinic, Bulgaria

Reviewed by:

Roxana Schillaci,

CONICET Instituto de Biología y

Medicina Experimental (IBYME),

Argentina

Ke-Da Yu,

Fudan University, China

Li Zhu,

Shanghai Jiao Tong University, China

*Correspondence:

Hengqiang Zhao

zhaochewh@whu.edu.cn

Specialty section:

This article was submitted to

Women's Cancer,

a section of the journal

Frontiers in Oncology

Received: 19 December 2020

Accepted: 30 April 2021

Published: 17 May 2021

Citation:

Zhao H and Gong Y (2021) The

Prognosis of Single Hormone Receptor-Positive Breast Cancer

Stratified by HER2 Status.

Front. Oncol. 11:643956.

doi: 10.3389/fonc.2021.643956
Single estrogen receptor $(\mathrm{ER})+$ and progesterone receptor $(\mathrm{PR})+$ tumors account for about $10 \%$ of all breast cancers. However, the prognosis of these single hormone receptor-positive $(\mathrm{HR}+)$ tumor remains unclear. We aimed to investigate the characteristics of single HR+ breast tumors according to HER2 status in order to improve the treatment of patients with single HR+. Patients from the SEER program (2010-2016) were divided into ER+PR-, ER-PR+, ER+PR+ and ER-PR- molecular subtypes stratified by HER2 status. Overall survival (OS) and breast cancer-specific survival (BCSS) were compared by Kaplan-Meier curves after propensity score matching (PSM). A total of 203,406 patients were enrolled. Single $E R+$ and PR+ tumors account for $11.9 \%$ of the total population. For HER2- subtype, patients with ER+PR- ( $n=16906$ pairs) and ER-PR+ ( $n=1395$ pairs) had worse prognoses than those with ER+PR+ with hazard ratio $(\mathrm{HR})$ and 95\% confidence interval $(\mathrm{Cl})$ of 1.52 (1.41-1.64) and 2.25 (1.76-2.88) for OS; and 1.94 (1.76-2.14) and 2.57 (1.94-3.40) for BCSS, respectively; ER+PR- showed a better prognosis than ER-PR+ $(n=1394$ pairs) and ER-PR- $(n=9626$ pairs) with HR (95\% $\mathrm{Cl})$ of 1.32 (1.06-1.65) and 1.44 (1.33-1.55) for OS, and 1.32 (1.03-1.69) and 1.46 (1.341.60) for BCSS, respectively; ER-PR+ had a similar prognosis relative to ER-PR- $(n=1395$ pairs) after PSM. For HER2+ subtype, patients with ER-PR+, ER+PR-, and ER-PR- had similar OS and BCSS; ER+PR+ showed a similar prognosis compare with ER-PR+ $(n=$ 535 pairs), but had better OS and BCSS than ER+PR- ( $n=5376$ pairs) and ER-PR- ( $n=$ 8143 pairs) after PSM. In addition, ER+PR+HER2+ showed similar OS and better BCSS compared with ER+PR+HER2- after PSM. In conclusion, single PR+ patients experienced poorer prognoses than single ER+ patients, and may be treated as ER-PR-patients in HER2- subtype. In HER2+ patients, both single ER + and single PR+ cases showed similar prognoses compared with ER-PR- cases, and may be treated as ER-PR-patients.

Keywords: breast cancer, estrogen receptor, progesterone receptor, HER2, prognosis 


\section{INTRODUCTION}

Breast cancer is a heterogeneous malignancy that can be divided into several molecular subtypes according to estrogen receptor (ER), progesterone receptor (PR), and human epidermal growth factor 2 (HER2). ER and PR are termed as hormone receptor (HR). The four main molecular subtypes are luminal A, luminal B, HER2+, and triple-negative breast cancer. This classification provides a good biomarker for prognosis and basis for targeted therapies.

Single ER+ and PR+ subtypes accounted for about $10 \%$ of all molecular subtypes of breast cancer (1). Some studies found that there were no differences in prognosis between ER+PR- and ER$\mathrm{PR}+$ patients $(2,3)$. Others found that ER-PR+ patients had worse prognosis compared with ER+PR- cases $(1,4)$. Research from the National Cancer Database and the Surveillance, Epidemiology, and End Results (SEER) program showed the same conclusion that single $\mathrm{HR}+$ tumors had worse prognosis than $\mathrm{ER}+\mathrm{PR}+$ tumors, and ER-PR+ subtype had similar prognosis relative to ER-PR- $(5,6)$. However, HER2 status, a vital predictor of breast cancer prognosis, was not included for analysis in these studies. Recently, a single-center study found that the clinicopathologic characteristics and prognosis were similar between $\mathrm{ER}+\mathrm{PR}+, \mathrm{ER}-\mathrm{PR}+$, and $\mathrm{ER}+\mathrm{PR}-$ in HER2+ patients (7). Due to the limited sample size of single $\mathrm{HR}+$ cases and the imbalance between groups, they obtained a meaningless and wide confidence interval and this may weaken the reliability of the results (7). To our knowledge, studies on the prognosis of single ER+ and PR+ stratified by HER2 status are very limited.

In order to discern and treat patients with single $\mathrm{HR}+$ better, we compared the prognosis between single ER+ and PR+ tumors, and compared them with the other molecular subtypes stratified by HER2 status. Unlike previous studies, we for the first time balanced the variables between groups using propensity score matching (PSM), and we found some novel results of the prognosis of single HR+ tumors after introducing HER2.

\section{PATIENTS AND METHODS}

\section{Ethics Statement}

The study population were obtained from SEER database from 2010 to 2016 (SEERStat user name: 10561-Nov2019). This study was granted an exemption from institutional review board approval for its deidentified information in a public database.

\section{Study Population}

ER and PR status were determined by immunohistochemistry. If $1 \%$ or greater cells stain positive, the test results are considered positive. HER2 status was available from 2010 in SEER database, we thus enroll patients from 2010. The following clinicopathologic characteristics were extracted from the database: patient age, year of diagnosis, race, marital status, insurance, pathological types, tumor grade, tumor stage,
T/N/M stage, number of metastatic axillary lymph nodes, surgery, radiation, chemotherapy, overall survival (OS), breast cancer-specific survival (BCSS), and survival months. Marital status comprises single, married, divorced/separated/widowed (DSW) and other. The pathology was categorized into invasive ductal carcinoma, invasive lobular carcinoma, and invasive ductal and lobular carcinoma. Poorly differentiated and anaplastic histology were defined as grade III. Tumor stage and T/N/M stage was based on American Joint Committee on Cancer (AJCC) stage of the 6th edition. Surgery was categorized into partial mastectomy, total mastectomy, and modified radical mastectomy. Only female patients with one primary tumor, positive histology, and age $\geq 18$ years were enrolled. Data with unknown information were excluded.

\section{Statistical Analysis}

Category variables were analyzed by $\chi^{2}$ test. Age and year of diagnosis were analyzed with Mann-Whitney $U$ test. The number of metastatic axillary lymph nodes was compared by Student's $t$ test. OS was defined as the duration between the date of initial diagnosis to death from any causes or last follow up. BCSS was defined as the time from diagnosis to death from breast cancer. The Kaplan-Meier curves of OS and BCSS were analyzed by log-rank test. Multivariable Cox proportional hazards regression was established to estimate hazard ratio (HR) and a 95\% confidence interval (CI) for OS and BCSS.

To minimize the imbalance of the variables between groups, PSM was performed using R software (ver. 3.3.3, https://www.rproject.org/) of package 'MatchIt'. Age, year of diagnosis, race, marital status, insurance, histology, grade, tumor stage, T/N/M stage, metastatic axillary lymph nodes, surgery, radiation, and chemotherapy were matched between groups. One-to-one matching with a caliper of 0.1 was used to balance the demographic, pathologic and treatment covariates as previously described (8). Other statistical analyses were performed with Stata/MP (ver. 14.2, StataCorp., College Station, TX), and GraphPad Prism (ver. 7.0, GraphPad Software, Inc). A two-sided $P$ value $<0.05$ was considered statistically different.

\section{RESULTS}

\section{Clinicopathologic Characteristics of the Study Population}

The flowchart of selection process was shown in Supplementary

Figure 1. A total of 203406 patients were included, including 133662 patients $(65.7 \%)$ for ER+PR+HER2-, 16906 (8.3\%) for ER+PR-HER2-, 1395 (0.7\%) for ER-PR+HER2-, 21439 (10.5\%) for ER-PR-HER2-; and 15646 (7.7\%) for ER+PR+HER2+, 5381 (2.6\%) for ER+PR-HER2+, 537 (0.3\%) for ER-PR+HER2+, and 8440 (4.1\%) for ER-PR-HER2+. The median follow-up duration of the study population was 35 months (range: 1-83 months). The clinicopathologic characteristics of each subtype were summarized in Table 1. 
TABLE 1 | Clinicopathologic characteristics of the study population $(n=203,406)$.

\begin{tabular}{|c|c|c|c|c|c|c|c|c|}
\hline \multirow[t]{2}{*}{ Category } & \multicolumn{4}{|c|}{ HER2- } & \multicolumn{4}{|c|}{ HER2+ } \\
\hline & $\begin{array}{c}\text { ER-PR+ } \\
(n=1395)\end{array}$ & $\begin{array}{c}\text { ER+PR- } \\
(n=16906)\end{array}$ & $\begin{array}{c}\text { ER-PR- } \\
(n=21439)\end{array}$ & $\begin{array}{c}\text { ER+PR+ } \\
(n=133662)\end{array}$ & $\begin{array}{l}\text { ER-PR+ } \\
(n=537)\end{array}$ & $\begin{array}{c}\text { ER+PR- } \\
(n=5381)\end{array}$ & $\begin{array}{c}\text { ER-PR- } \\
(n=8440)\end{array}$ & $\begin{array}{c}\text { ER+PR+ } \\
(n=15646)\end{array}$ \\
\hline Age (year) & $57(48-67)$ & $62(54-70)$ & 55 (47-65) & 61 (51-69) & $56(47-65)$ & 58 (51-66) & $56(48-65)$ & $56(46-65)$ \\
\hline $\begin{array}{l}\text { Year of } \\
\text { diagnosis } \\
\text { Race }\end{array}$ & $\begin{array}{c}2013 \\
(2011-2015)\end{array}$ & $\begin{array}{c}2013 \\
(2011-2015)\end{array}$ & $\begin{array}{c}2013 \\
(2011-2015)\end{array}$ & $\begin{array}{c}2013 \\
(2011-2015)\end{array}$ & $\begin{array}{c}2013 \\
(2012-2015)\end{array}$ & $\begin{array}{c}2013 \\
(2011-2015)\end{array}$ & $\begin{array}{c}2013 \\
(2011-2015)\end{array}$ & $\begin{array}{c}2013 \\
(2011-2015)\end{array}$ \\
\hline White & $1021(73.2)$ & 13095 (77.5) & 15403 (71.8) & 109651 (82.0) & 395 (73.6) & 4079 (75.8) & 6055 (71.7) & $12144(77.6)$ \\
\hline Black & 270 (19.4) & 2196 (13.0) & $4366(20.4)$ & $10889(8.1)$ & 70 (13.0) & 645 (12.0) & 1187 (14.1) & $1736(11.1)$ \\
\hline \multicolumn{9}{|l|}{ Insurance } \\
\hline Uninsured & 25 (1.8) & 217 (1.3) & 417 (1.9) & 1675 (1.3) & $10(1.9)$ & $88(1.6)$ & $152(1.8)$ & $263(1.7)$ \\
\hline \multicolumn{9}{|l|}{ Marital status } \\
\hline Single & 231 (16.6) & 2413 (14.3) & 3571 (16.7) & 18758 (14.0) & 71 (13.2) & 837 (15.6) & 1290 (15.3) & 2635 (16.8) \\
\hline Married & 796 (57.1) & $9424(55.7)$ & $11941(55.7)$ & 78702 (58.9) & $322(60.0)$ & 3122 (58.0) & 4997 (59.2) & 9298 (59.4) \\
\hline DSW & 308 (22.1) & 4369 (25.8) & $4944(23.1)$ & 30837 (23.1) & 118 (22.0) & 1203 (22.4) & 1796 (21.3) & 3111 (19.9) \\
\hline \multicolumn{8}{|l|}{ Grade } & 602 (3.8) \\
\hline I & $21(1.5)$ & 3415 (20.2) & 308 (1.4) & 40471 (30.3) & $6(1.1)$ & $236(4.4)$ & $120(1.4)$ & 1123 (7.2) \\
\hline$\|$ & 218 (15.6) & 7147 (42.3) & 3476 (16.2) & $70474(52.7)$ & 125 (23.3) & 1984 (36.9) & 1924 (22.8) & $6860(43.8)$ \\
\hline \multicolumn{9}{|l|}{ Pathology } \\
\hline IDC & 1350 (96.8) & 13397 (79.2) & 20920 (97.6) & 107194 (80.2) & 522 (97.2) & 5015 (93.2) & 8271 (98.0) & 14222 (90.9) \\
\hline ILC & $19(1.4)$ & 2501 (14.8) & $222(1.0)$ & 16067 (12.0) & $2(0.4)$ & 175 (3.3) & $70(0.8)$ & 657 (4.2) \\
\hline IDLC & $26(1.9)$ & 1008 (6.0) & $297(1.4)$ & 10401 (7.8) & $13(2.4)$ & $191(3.5)$ & $99(1.2)$ & 767 (4.9) \\
\hline \multicolumn{9}{|l|}{ Stage } \\
\hline 1 & 531 (38.1) & 8453 (50.0) & 8414 (39.2) & 78791 (58.9) & $194(36.1)$ & 2324 (43.2) & 3232 (38.3) & 6913 (44.2) \\
\hline$\|$ & 681 (48.8) & 6107 (36.1) & 9633 (44.9) & 41854 (31.3) & 243 (45.3) & 2161 (40.2) & 3480 (41.2) & $6322(40.4)$ \\
\hline III & $161(11.5)$ & 2090 (12.4) & 2985 (13.9) & 11891 (8.9) & $88(16.4)$ & 754 (14.0) & 1481 (17.5) & 2124 (13.6) \\
\hline IV & $22(1.6)$ & 256 (1.5) & 407 (1.9) & $1126(0.8)$ & $12(2.2)$ & $142(2.6)$ & 247 (2.9) & 287 (1.8) \\
\hline \multicolumn{9}{|l|}{ Tumor } \\
\hline T1 & 633 (45.4) & 9704 (57.4) & 9862 (46.0) & 90610 (67.8) & 252 (46.9) & $2822(52.4)$ & 4019 (47.6) & 8337 (53.3) \\
\hline T2 & 621 (44.5) & 5663 (33.5) & 9159 (42.7) & 35490 (26.6) & 217 (40.4) & 1993 (37.0) & 3296 (39.1) & 5879 (37.6) \\
\hline T3 & $89(6.4)$ & 1135 (6.7) & 1625 (7.6) & $5869(4.4)$ & $40(7.4)$ & 391 (7.3) & 727 (8.6) & 969 (6.2) \\
\hline $\mathrm{T} 4$ & $52(3.7)$ & $404(2.4)$ & 793 (3.7) & 1693 (1.3) & $28(5.2)$ & 175 (3.3) & $398(4.7)$ & $461(2.9)$ \\
\hline \multicolumn{9}{|l|}{ Node } \\
\hline NO & $954(68.4)$ & 11583 (68.5) & $14458(67.4)$ & $94487(70.7)$ & 320 (59.6) & 3398 (63.1) & 5069 (60.1) & 9799 (62.6) \\
\hline N1 & 328 (23.5) & 3682 (21.8) & $4732(22.1)$ & $29698(22.2)$ & $156(29.1)$ & 1373 (25.5) & 2230 (26.4) & 4190 (26.8) \\
\hline N2 & $62(4.4)$ & 1014 (6.0) & 1355 (6.3) & 6467 (4.8) & $42(7.8)$ & $363(6.7)$ & $653(7.7)$ & 1087 (6.9) \\
\hline N3 & $51(3.7)$ & $627(3.7)$ & 894 (4.2) & 3010 (2.3) & 19 (3.5) & 247 (4.6) & 488 (5.8) & 570 (3.6) \\
\hline $\begin{array}{l}\text { ALNM } \\
\text { Metastasis }\end{array}$ & $1.04 \pm 3.39$ & $1.18 \pm 3.38$ & $1.19 \pm 3.39$ & $0.92 \pm 2.68$ & $1.33 \pm 3.29$ & $1.23 \pm 3.17$ & $1.52 \pm 3.88$ & $1.24 \pm 3.19$ \\
\hline MO & 1373 (98.4) & 16650 (98.5) & 21032 (98.1) & 132536 (99.2) & $525(97.8)$ & 5239 (97.4) & 8193 (97.1) & 15359 (98.2) \\
\hline M1 & $22(1.6)$ & 256 (1.5) & 407 (1.9) & $1126(0.8)$ & $12(2.2)$ & $142(2.6)$ & 247 (2.9) & $287(1.8)$ \\
\hline \multicolumn{9}{|l|}{ Surgery } \\
\hline PM & 785 (56.3) & 9891 (58.5) & $11441(53.4)$ & $84530(63.2)$ & $240(44.7)$ & 2493 (46.3) & 3618 (42.9) & 8043 (51.4) \\
\hline TM & 370 (26.5) & 4192 (24.8) & 5712 (26.6) & $32232(24.1)$ & $180(33.5)$ & 1719 (31.9) & 2702 (32.0) & 4605 (29.4) \\
\hline MRM & $240(17.2)$ & $2823(16.7)$ & $4286(20.0)$ & 16900 (12.6) & $117(21.8)$ & 1169 (21.7) & $2120(25.1)$ & 2998 (19.2) \\
\hline \multicolumn{9}{|l|}{ Radiation } \\
\hline No & 561 (40.2) & 6687 (39.6) & 9307 (43.4) & $50080(37.5)$ & 246 (45.8) & 2610 (48.5) & 4174 (49.5) & 6909 (44.2) \\
\hline Yes & 834 (59.8) & $10219(60.4)$ & 12132 (56.6) & 83582 (62.5) & 291 (54.2) & 2771 (51.5) & 4266 (50.5) & 8737 (55.8) \\
\hline \multicolumn{9}{|c|}{ Chemotherapy } \\
\hline No & 351 (25.2) & 9122 (54.0) & 4740 (22.1) & 95852 (71.7) & 105 (19.6) & 1335 (24.8) & 1768 (20.9) & 3792 (24.2) \\
\hline Yes & $1044(74.8)$ & 7784 (46.0) & 16699 (77.9) & 37810 (28.3) & $432(80.4)$ & $4046(75.2)$ & $6672(79.1)$ & $11854(75.8)$ \\
\hline
\end{tabular}

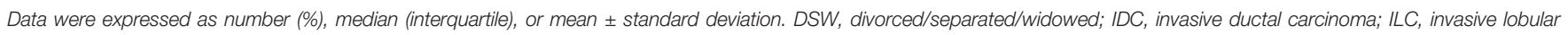

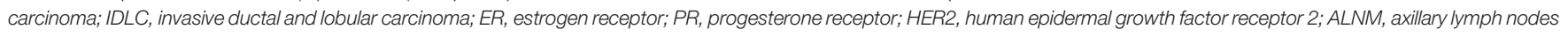
metastasis; PM, partial mastectomy; TM, total mastectomy; MRM; modified radical mastectomy.

\section{Predictors for OS and BCSS by Multivariate Cox Regression Analysis}

Compared with ER+PR+HER2-, patients with ER+PR-HER2-, ER-PR+HER2-, and ER-PR-HER2- were associated with compromised OS with HR (95\% CI) of 1.67 (1.58-1.77), 2.36 (2.02-2.75), and $2.72(2.59-2.87)$, respectively. In addition, ER+PR-HER2+ and ER-PR-HER2+ were associated with compromised OS compared with $\mathrm{ER}+\mathrm{PR}+\mathrm{HER} 2$ - with $\mathrm{HR}$ 
(95\% CI) of 1.20 (1.09-1.34) and 1.27 (1.17-1.38), respectively. However, patients with $\mathrm{ER}+\mathrm{PR}+\mathrm{HER} 2+$ showed marginally better OS than those with ER+PR+HER2-with HR (95\% CI) of 0.93 (0.86-1.01). No significant difference in OS was observed between patients with ER+PR+HER2- and ER-PR+HER2+ (Table 2). BCSS showed the same trend as OS. In addition, patients with $\mathrm{ER}+\mathrm{PR}+\mathrm{HER} 2+$ showed improved BCSS compared with ER+PR+HER2- cases with HR (95\% CI) of 0.89 (0.80-0.99) (Supplementary Table 1).

\section{Kaplan-Meier Curves of OS and BCSS Before PSM}

For HER2- subtype, ER+PR+ had better OS compared with $\mathrm{ER}+\mathrm{PR}-, \mathrm{ER}-\mathrm{PR}+$, and ER-PR- $(\log$-rank $p<0.001$ for all).

TABLE 2 | Overall survival by multivariate Cox proportional analysis.

\begin{tabular}{|c|c|c|}
\hline Category & HR (95\% Cl) & $P$-value \\
\hline Age & $1.038(1.036-1.039)$ & $<0.001$ \\
\hline Year of diagnosis & $0.98(0.96-0.99)$ & $<0.001$ \\
\hline \multicolumn{3}{|l|}{ Race (vs White) } \\
\hline Black & $1.23(1.17-1.29)$ & $<0.001$ \\
\hline Other & $0.71(0.66-0.77)$ & $<0.001$ \\
\hline \multicolumn{3}{|l|}{ Insurance (vs uninsured) } \\
\hline Insured & $0.82(0.71-0.93)$ & 0.003 \\
\hline \multicolumn{3}{|l|}{ Marital status (vs single) } \\
\hline Married & $0.73(0.69-0.77)$ & $<0.001$ \\
\hline Divorced/separated/widowed & $1.01(0.96-1.07)$ & 0.653 \\
\hline Other & $0.84(0.76-0.92)$ & $<0.001$ \\
\hline \multicolumn{3}{|l|}{ Grade (vs I) } \\
\hline$\|$ & $1.24(1.16-1.32)$ & $<0.001$ \\
\hline III-IV & $1.88(1.76-2.01)$ & $<0.001$ \\
\hline \multicolumn{3}{|l|}{ Pathology (vs invasive ductal carcinoma) } \\
\hline Invasive lobular carcinoma & $0.86(0.80-0.92)$ & $<0.001$ \\
\hline Invasive ductal and lobular carcinoma & $0.91(0.84-0.98)$ & 0.015 \\
\hline \multicolumn{3}{|l|}{ Tumor (vs T1) } \\
\hline T2 & $1.72(1.65-1.80)$ & $<0.001$ \\
\hline Т3 & $2.53(2.37-2.70)$ & $<0.001$ \\
\hline $\mathrm{T} 4$ & $3.15(2.92-3.40)$ & $<0.001$ \\
\hline \multicolumn{3}{|l|}{ Node (vs NO) } \\
\hline N1 & $1.72(1.65-1.80)$ & $<0.001$ \\
\hline N2 & $2.53(2.37-2.70)$ & $<0.001$ \\
\hline N3 & $3.15(2.92-3.40)$ & $<0.001$ \\
\hline Axillary lymph nodes metastasis & $1.03(1.02-1.04)$ & $<0.001$ \\
\hline \multicolumn{3}{|l|}{ Metastasis (vs MO) } \\
\hline M1 & $2.90(2.70-3.11)$ & $<0.001$ \\
\hline \multicolumn{3}{|l|}{ Molecular subtypes (vs ER+PR+HER2-) } \\
\hline ER+PR-HER2- & $1.67(1.58-1.77)$ & $<0.001$ \\
\hline ER-PR+HER2- & $2.36(2.02-2.75)$ & $<0.001$ \\
\hline ER-PR-HER2- & $2.72(2.59-2.87)$ & $<0.001$ \\
\hline $\mathrm{ER}+\mathrm{PR}+\mathrm{HER} 2+$ & $0.93(0.86-1.01)$ & 0.071 \\
\hline ER+PR-HER2+ & $1.20(1.09-1.34)$ & $<0.001$ \\
\hline ER-PR+HER2+ & $1.04(0.74-1.46)$ & 0.805 \\
\hline ER-PR-HER2+ & $1.27(1.17-1.38)$ & $<0.001$ \\
\hline \multicolumn{3}{|l|}{ Surgery (vs partial mastectomy) } \\
\hline Total mastectomy & $1.02(0.96-1.07)$ & 0.584 \\
\hline Modified radical mastectomy & $1.22(1.16-1.28)$ & $<0.001$ \\
\hline \multicolumn{3}{|l|}{ Radiation (vs no) } \\
\hline Yes & $0.66(0.63-0.68)$ & $<0.001$ \\
\hline \multicolumn{3}{|l|}{ Chemotherapy (vs no) } \\
\hline Yes & $0.73(0.70-0.76)$ & $<0.001$ \\
\hline
\end{tabular}

$H R$, hazard ratio; $\mathrm{Cl}$, confidence interval; $E R$, estrogen receptor; $P R$, progesterone receptor.
In addition, ER+PR- showed better OS than ER-PR+ and ER-PR- (log-rank $p<0.001$ for both). Additionally, ER-PR+ showed better OS than ER-PR- $(\log$-rank $p=0.021)$ before PSM (Figures 1A, B).

For HER2+ subgroup, ER+PR+ showed better OS than $\mathrm{ER}+\mathrm{PR}$ - and ER-PR- (log-rank $p<0.001$ for both), while ER $+\mathrm{PR}+$ had similar OS relative to ER-PR+ $(\log$-rank $p=0.085)$. In addition, ER+PR- showed better OS than ER-PR- $(\log$-rank $p=$ 0.024). However, no significant difference in OS was observed between ER+PR- and ER-PR+ $(\log -$ rank $p=0.370)$. What's more, ER-PR+ showed similar OS compared with ER-PR( $\log$-rank $p=0.082$ ) (Figures 1A, B). BCSS showed the same trend as OS. In addition, ER-PR+ patients showed better BCSS than ER-PR- patients before PSM (log-rank $p=0.045$ ) (Supplementary Figure 2).

\section{Kaplan-Meier Curves of OS and BCSS After PSM}

To minimize the imbalance of baseline clinicopathologic characteristics between groups, PSM was performed. The absolute values of standardized differences of the matched variables were $<0.1$, indicating that the variables were well balanced between groups after matching. In addition, the statistical differences in the baseline characteristics between groups were reduced after PSM.

For HER2- subgroup, there were no differences in OS between ER-PR+ and ER-PR- with HR (95\% CI) of 0.95 (0.77-1.18) in a matched cohort of 1395 paired cases (Figure 2A and Supplementary Table 2). However, ER+PR- showed better OS than ER-PR- with HR (95\% CI) of 1.44 (1.31-1.55) in a matched cohort of 9626 paired cases (Figure 2B and Supplementary Table 3). Patients with ER+PR- showed better OS than patients with ER-PR+ $(n=1394$ pairs $)$ with $\mathrm{HR}$ (95\% CI) of 1.32 (1.06-1.65) (Figure 2C and Supplementary Table 4). ER+PR+ showed better OS than ER+PR- with HR (95\% CI) of 1.52 (1.41-1.64) ( $n=16906$ pairs) (Figure 2D; Supplementary Table 5), and better OS than ER-PR+ $(n=1395$ pairs $)$ with HR (95\% CI) of 2.25 (1.76-2.88) (Figure 2E and Supplementary Table 6). We further found that there was no difference in OS between $\mathrm{ER}+\mathrm{PR}+\mathrm{HER} 2+$ and $\mathrm{ER}+\mathrm{PR}+\mathrm{HER} 2$-with $\mathrm{HR}$ (95\% CI) of $0.99(0.89-1.09)(n=15640$ pairs) (Figure $2 \mathbf{F}$ and Supplementary Table 7). BCSS showed the same trend as OS. However, patients with $\mathrm{ER}+\mathrm{PR}+\mathrm{HER} 2+$ predicted better BCSS than those with ER+PR+HER2- with HR (95\% CI) of 0.86 (0.76-0.98) (Supplementary Figure 3).

For HER2+ subgroup, patients with ER-PR- showed similar OS compared with ER-PR+ $(n=536$ pairs) (Figure $3 \mathbf{A}$ and Supplementary Table 8), and ER+PR- ( $n=4984$ pairs) (Figure 3B and Supplementary Table 9). ER+PR+ showed better OS than ER-PR- with HR (95\% CI) of $1.37(1.22-1.44)(n=8143$ pairs) (Figure 3C and Supplementary Table 10). No significant differences in OS were observed between patient with $\mathrm{ER}+\mathrm{PR}+$ and ER-PR+ ( $n=535$ pairs) (Figure 3D and Supplementary Table 11). Patients with $\mathrm{ER}+\mathrm{PR}+$ had better OS than patients with ER+PR- with HR (95\% CI) of 1.26 (1.09-1.45) $(n=5376$ pairs) (Figure 3E and Supplementary Table 12). There was no significant difference in OS between patients with ER+PR- and 


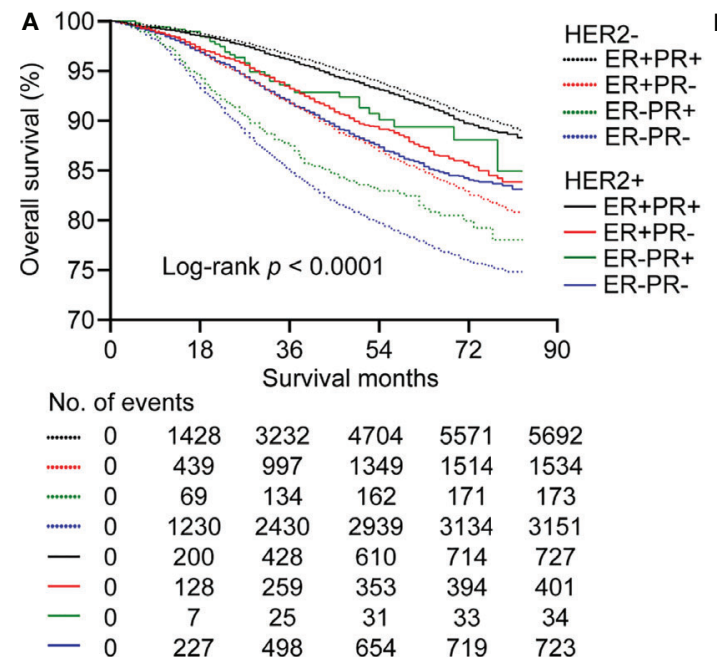

\begin{tabular}{|c|c|c|c|}
\hline \multicolumn{4}{|c|}{ B HER2- subgroup } \\
\hline ER+PR+ & & & \\
\hline$p<0.001$ & ER+PR- & & \\
\hline$p<0.001$ & $p<0.001$ & ER-PR+ & \\
\hline$p<0.001$ & $p<0.001$ & $p=0.021$ & ER-PR- \\
\hline \multicolumn{4}{|c|}{ Log-rank test between groups } \\
\hline \multicolumn{4}{|c|}{ HER2+ subgroup } \\
\hline \multicolumn{4}{|c|}{\begin{tabular}{|l|}
$\mathrm{ER}+\mathrm{PR}+$ \\
\end{tabular}} \\
\hline$p<0.001$ & $\mathrm{ER}+\mathrm{PR}-$ & & \\
\hline$p=0.085$ & $p=0.370$ & ER-PR+ & \\
\hline$p<0.001$ & $p=0.024$ & $p=0.082$ & ER-PR- \\
\hline
\end{tabular}

FIGURE 1 | Overall survival of patients stratified by estrogen receptor (ER), progesterone receptor (PR) and human epidermal growth factor Receptor 2 (HER2) status before propensity score matching. (A) Kaplan-Meier survival curves of overall survival. (B) League table of comparison by log-rank test.

ER-PR+ with HR (95\% CI) of $1.10(0.68-1.80)$ ( $n=537$ pairs) (Figure 3F and Supplementary Table 13). BCSS showed the same trend as OS (Supplementary Figure 4).

\section{DISCUSSION}

In this study, we found that single $\mathrm{HR}+$ showed different effect on the prognosis of HER2- and HER2+ breast tumors. ER+PR- had better prognosis than ER-PR+ in HER2- cases. However, ER+PRshowed similar prognosis compared with ER-PR+ in HER2+ cases. In addition, both ER+PR- and ER-PR+ cases had higher overall mortality than $\mathrm{ER}+\mathrm{PR}+$ patients in HER2- subtype. For HER2+ subtype, ER-PR+ showed similar prognosis compared with $\mathrm{ER}+\mathrm{PR}+\mathrm{ER}+\mathrm{PR}+\mathrm{HER} 2+$ patients had similar OS and better BCSS relative to those with $\mathrm{ER}+\mathrm{PR}+\mathrm{HER} 2-$.

A recent study found that ER+PR- and ER-PR+ had similar disease-free survival and OS compared with ER-PR- in both HER2+ and HER2- subgroups (7). However, we found that $\mathrm{ER}+\mathrm{PR}$ - showed better OS and BCSS than ER-PR- in HER2subgroup. This inconsistence might result from the sample size, statistical methods, and the adjusted variables. In addition, this study failed to compared the survival difference between $\mathrm{ER}+\mathrm{PR}$ - and ER-PR+ (7). We found that there were no differences in the prognoses between ER+PR- and ER-PR+ and ER-PR- after PSM in HER2+ subgroup. This indicated that patients with single $\mathrm{ER}+$ or $\mathrm{PR}+$ may gain limited survival benefit from endocrine therapy when facing HER2+. For the limited sample size, they failed to fully investigate the survival differences of ER+PR+ $(n=518)$ and ER-PR+ $(n=30)$ and $\mathrm{ER}+\mathrm{PR}-(n=159)$ in HER2+ subtype. We found that patients with $\mathrm{ER}+\mathrm{PR}+(n=15646)$ showed similar prognosis relative to those with ER-PR+ $(n=537)$, but had better prognosis than patients with ER+PR- $(n=5381)$ and ER-PR- $(n=8840)$ in HER2+ subtype after PSM.

The clinicopathologic characteristics were highly consistent between ER-PR+HER2+ and ER-PR-HER2+. However, patients with ER+PR-HER2+ were older, had lower tumor grade and tumor stage compared with ER-PR-HER2+. It seemed that patients with ER-PR+HER2+ were more likely to gain survival benefit from endocrine therapy or HER2-targeted therapy than those with ER + PR-HER2+. It indicated that PR levels may reflect growth factor activity within a tumor. Low or absent PR expression in some tumors indicated high HER2 activity. The increased growth factor signaling may reduce the ability of tamoxifen to act as an antagonist, resulting in selective ER modulator resistance (9).

Unlike the role of ER+PR- in HER2+ tumors, patients with $\mathrm{ER}+\mathrm{PR}$ - showed better prognoses relative to those with ER-PR+ and ER-PR- in HER2- subtype after PSM. We found that ER-PR+HER2- showed similar prognosis compared with ERPR-HER2-. In addition, ER+PR+HER2- showed better OS and BCSS than ER+PR-HER2-. Considering the survival differences between ER+PR- and ER-PR- in HER2+ and HER2- subtypes, we can speculate that there existed a signaling crosstalk between ER/PR and HER2. Growth factor can directly modulate ER activity via phosphorylation of ER itself or via phosphorylation of coregulators, it also downregulated PR levels independent of ER levels or activity (9). The proportion of ER+PR- and ER-PR+ tumors was $11.9 \%$ of in this study. ER and PR status can change during breast cancer development $(10,11)$. Therefore, the conversion from $\mathrm{ER}+\mathrm{PR}+$ to single $\mathrm{ER}+$ or $\mathrm{PR}+$, or to $\mathrm{ER}-\mathrm{PR}$ may indicate disease progression.

The proportion of ER-PR+ phenotype represents $1.0 \%$ of the total patients in our study. A study re-evaluated 43 of 2432 $(1.8 \%)$ patients reported as ER-PR+ in a reference laboratory. However, none of the cases were the initial ER-PR+ (12). Another study re-evaluated 27 of 9844 (0.3\%) patients which 

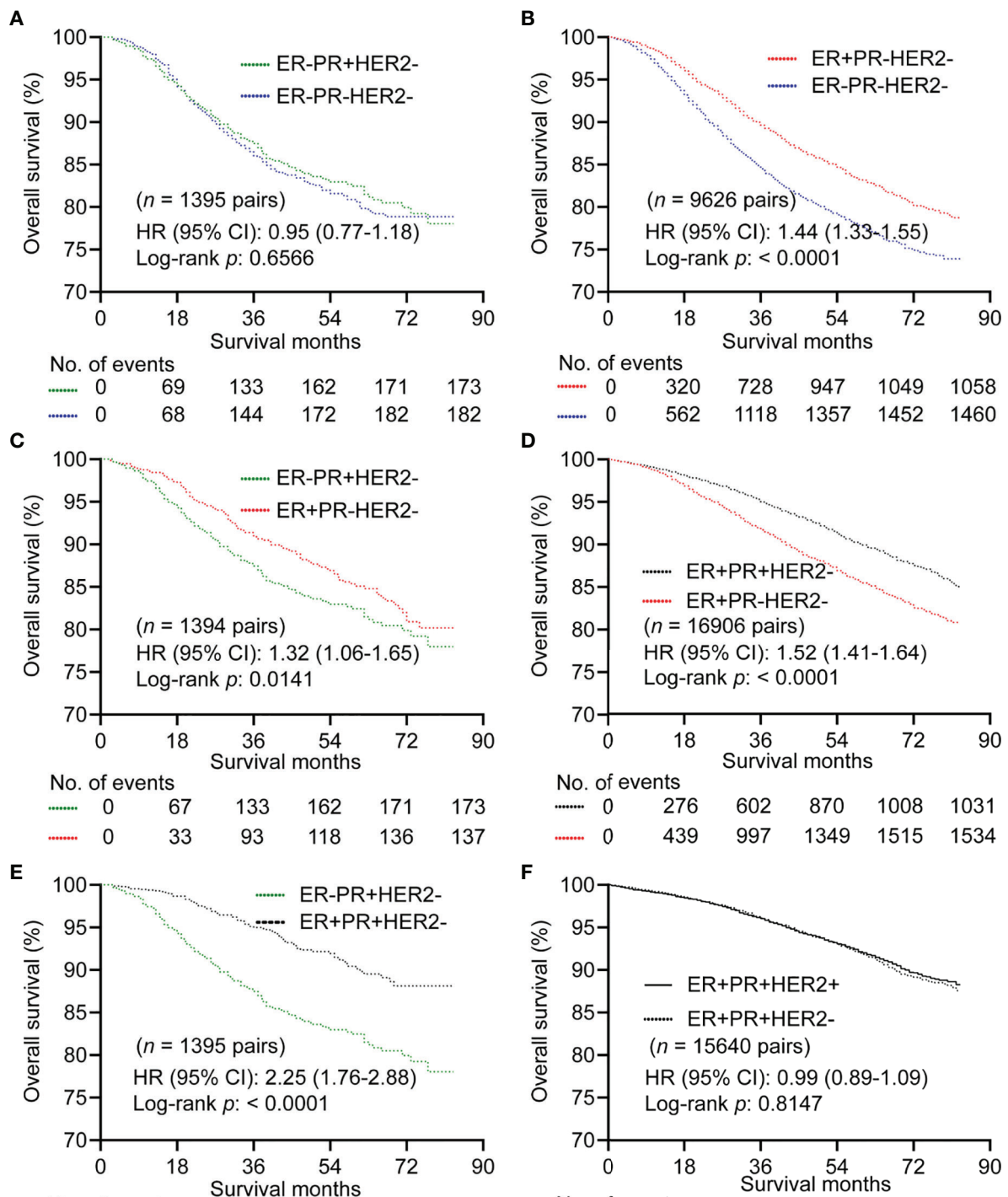

F

\begin{tabular}{rrrcccc}
\multicolumn{2}{c}{ No. of events } & & & & & \\
........ & 0 & 69 & 133 & 162 & 171 & 173 \\
$\ldots . .$. & 0 & 16 & 50 & 69 & 81 & 81
\end{tabular}

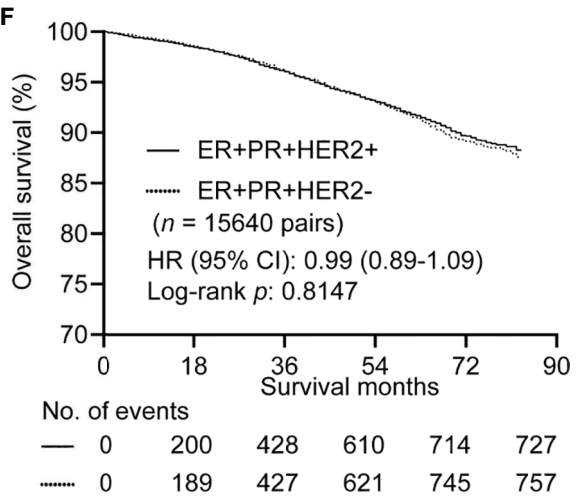

FIGURE 2 | Kaplan-Meier curves of overall survival after propensity score matching in HER2- subgroup. (A) Comparison between ER-PR+ and ER-PR-. (B) Comparison between ER+PR- and ER-PR-. (C) Comparison between ER-PR+ and ER+PR-. (D) Comparison between ER+PR+ and ER+PR-. (E) Comparison between ER+PR+ and ER-PR+. (F) Comparison between ER+PR+HER2- and ER+PR+HER2+. ER, estrogen receptor; PR, progesterone receptor; HER2, human epidermal growth factor Receptor 2.

were initially diagnosed with ER-PR+ breast carcinoma, and 7 patients remained ER-PR+ (13). However, another study found that breast carcinoma of ER-PR+ existed, although rare $(1.1 \%$ of all phenotypes), and had distinct clinicopathologic characteristics (14). The status of ER-PR+ should be evaluated carefully to avoid technical artifacts (15).

Contrary to our conventional thought that ER+PR+HER2predicted the best survival, and a low risk of local or regional recurrence $(16,17)$, we found that patients with ER+PR+HER2+ showed similar OS, and even better BCSS relative to those with $\mathrm{ER}+\mathrm{PR}+\mathrm{HER} 2-$. A previous study showed that HR+HER2+ subtype experienced the better BCSS than those with HR+HER2- for stage IV breast cancer (16). The introduction of trastuzumab therapy may account for the improved prognosis of HER2+ patients. Trastuzumab plus adjuvant chemotherapy was associated with a $33 \%$ reduction in the risk of mortality compared with chemotherapy alone for operable HER2+ breast cancer (18). In CLEOPATRA trial, dual inhibition of HER2 signaling with pertuzumab and trastuzumab significantly improved OS compared with trastuzumab alone for HER2+ metastatic breast cancer (median OS: 56.5 months vs 40.8 months) (19). With the development of HER2 inhibitors, 

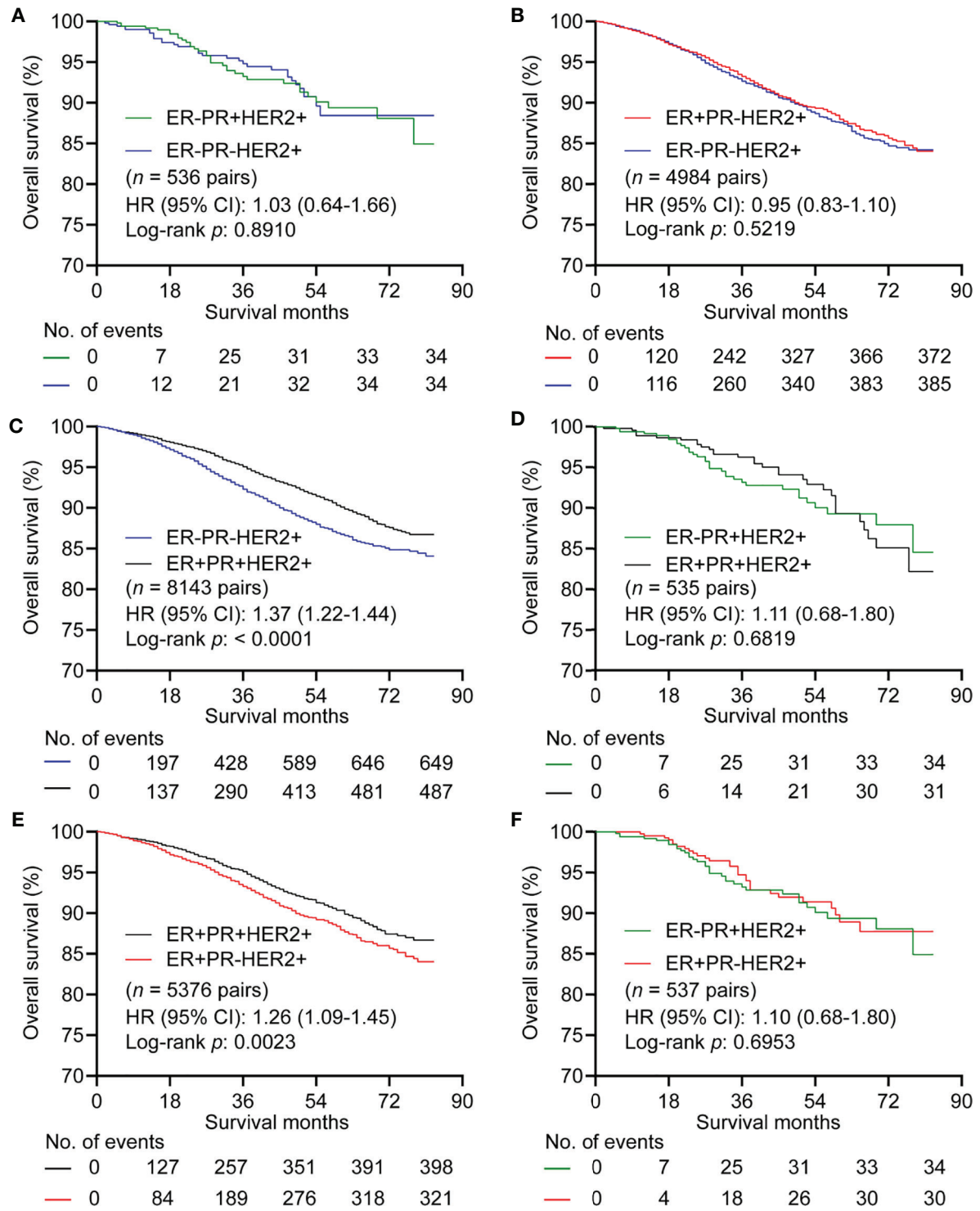

FIGURE 3 | Kaplan-Meier curves of overall survival in HER2+ subgroup after propensity score matching. (A) Comparison between ER-PR+ and ER-PR-. (B) Comparison between ER+PR- and ER-PR-. (C) Comparison between ER-PR- and ER+PR+. (D) Comparison between ER+PR+ and ER-PR+. (E) Comparison between ER+PR+ and ER+PR-. (F) Comparison between ER+PR- and ER-PR+. ER, estrogen receptor; PR, progesterone receptor; HER2, human epidermal growth factor Receptor 2.

patients with $\mathrm{ER}+\mathrm{PR}+\mathrm{HER} 2+$ may gain better prognoses than those with $\mathrm{ER}+\mathrm{PR}+\mathrm{HER} 2-$.

The limitations of this study must be acknowledged. First, the selection bias was evitable for the retrospective design. However, the statistical differences in baseline variables were significantly reduced after performing PSM, which may improve the reliability of the results. In addition, the endocrine and HER2 targeted therapies were not recorded in the database. Therefore, they were not included for analysis. Additionally, the exact expression levels of ER and PR were unavailable. We can't re-evaluate single $\mathrm{ER}+$ and $\mathrm{PR}+$ status.
In conclusion, this is the largest known study investigating the prognosis of breast cancer stratified by ER, PR and HER2 status. We found that single ER+ and PR+ subtypes showed different roles in the prognosis of breast cancer according to HER2 status. For HER2- subgroup, ER+PR+ showed the best prognosis, followed by ER+PR-, and worst for ER-PR+ and ER-PRsubtypes. For HER2+ subgroup, ER+PR+ showed similar prognosis relative to ER-PR+, but better prognosis than ER $+\mathrm{PR}$ - and ER-PR- subtypes. HER2 status didn't decrease the survival of patients with $\mathrm{ER}+\mathrm{PR}+$. Although we strictly included cases and balanced the variables between groups with a large 
population, the results should be cautiously interpreted when dealing with single ER+ or PR+ breast cancers.

\section{DATA AVAILABILITY STATEMENT}

The original contributions presented in the study are included in the article/Supplementary Material. Further inquiries can be directed to the corresponding author.

\section{AUTHOR CONTRIBUTIONS}

HZ: conception, data acquisition. HZ and YG: data analysis and drafting the article. HZ and YG: revised it critically for important intellectual content. HZ: investigation, project administration,

\section{REFERENCES}

1. Li Y, Yang D, Yin X, Zhang X, Huang J, Wu Y, et al. Clinicopathological Characteristics and Breast Cancer-Specific Survival of Patients With Single Hormone Receptor-Positive Breast Cancer. JAMA Netw Open (2020) 3: e1918160. doi: 10.1001/jamanetworkopen.2019.18160

2. Rakha EA, El-Sayed ME, Green AR, Paish EC, Powe DG, Gee J, et al. Biologic and Clinical Characteristics of Breast Cancer With Single Hormone Receptor Positive Phenotype. J Clin Oncol (2007) 25:4772-8. doi: 10.1200/JCO. 2007.12.2747

3. Shen T, Brandwein-Gensler M, Hameed O, Siegal GP, Wei S. Characterization of Estrogen Receptor-Negative/Progesterone ReceptorPositive Breast Cancer. Hum Pathol (2015) 46:1776-84. doi: 10.1016/ j.humpath.2015.07.019

4. Li Z, Tu Y, Wu Q, Wang Z, Li J, Zhang Y, et al. Clinical Characteristics and Outcomes of Single Versus Double Hormone Receptor-Positive Breast Cancer in 2 Large Databases. Clin Breast Cancer (2020) 20:e151-e63. doi: 10.1016/j.clbc.2019.07.002

5. Dauphine C, Moazzez A, Neal JC, Chlebowski RT, Ozao-Choy J. Single Hormone Receptor-Positive Breast Cancers Have Distinct Characteristics and Survival. Ann Surg Oncol (2020) 27:4687-94. doi: 10.1245/s10434-020-08898-5

6. Lv M, Mao Y, Song Y, Wang Y, Liu X, Wang X, et al. Clinical Features and Survival of Single Hormone Receptor-Positive Breast Cancer: A PopulationBased Study of 531,605 Patients. Clin Breast Cancer (2020) 20:e589-e99. doi: $10.1016 /$ j.clbc.2020.04.010

7. Bae SY, Kim S, Lee JH, Lee HC, Lee SK, Kil WH, et al. Poor Prognosis of Single Hormone Receptor- Positive Breast Cancer: Similar Outcome as Triple-Negative Breast Cancer. BMC Cancer (2015) 15:138. doi: 10.1186/ s12885-015-1121-4

8. Zhao H. The Prognosis of Invasive Ductal Carcinoma, Lobular Carcinoma and Mixed Ductal and Lobular Carcinoma According to Molecular Subtypes of the Breast. Breast Cancer (2020) 28:187-95. doi: 10.1007/s12282-02001146-4

9. Cui X, Schiff R, Arpino G, Osborne CK, Lee AV. Biology of Progesterone Receptor Loss in Breast Cancer and its Implications for Endocrine Therapy. J Clin Oncol (2005) 23:7721-35. doi: 10.1200/JCO.2005.09.004

10. Fujii K, Watanabe R, Ando T, Kousaka J, Mouri Y, Yoshida M, et al. Alterations in Three Biomarkers (Estrogen Receptor, Progesterone Receptor and Human Epidermal Growth Factor 2) and the Ki67 Index Between Primary and Metastatic Breast Cancer Lesions. BioMed Rep (2017) 7:53542. doi: 10.3892/br.2017.1003 and supervision. All authors contributed to the article and approved the submitted version.

\section{FUNDING}

This work was supported by the Fundamental Research Funds for the Central Universities (grant number 2042020kf0063).

\section{SUPPLEMENTARY MATERIAL}

The Supplementary Material for this article can be found online at: https://www.frontiersin.org/articles/10.3389/fonc. 2021.643956/full\#supplementary-material

11. Li MH, Hou CL, Wang C, Sun AJ. Her-2, ER, PR Status Concordance in Primary Breast Cancer and Corresponding Metastatic Lesion in Lymph Node in Chinese Women. Pathol Res Pract (2016) 212:252-7. doi: 10.1016/ j.prp.2015.11.019

12. Maleki Z, Shariat S, Mokri M, Atri M. ER-Negative /PR-Positive Breast Carcinomas or Technical Artifacts in Immunohistochemistry? Arch Iran Med (2012) 15:366-9. doi: 012156/AIM.0010

13. Kuroda H, Muroi N, Hayashi M, Harada O, Hoshi K, Fukuma E, et al. Oestrogen Receptor-Negative/Progesterone Receptor-Positive Phenotype of Invasive Breast Carcinoma in Japan: Re-Evaluated Using Immunohistochemical Staining. Breast Cancer (2019) 26:249-54. doi: 10.1007/s12282-018-0898-9

14. Ahmed SS, Thike AA, Zhang K, Lim JC, Tan PH. Clinicopathological Characteristics of Oestrogen Receptor Negative, Progesterone Receptor Positive Breast Cancers: Re-Evaluating Subsets Within This Group. J Clin Pathol (2017) 70:320-26. doi: 10.1136/jclinpath-2016-203847

15. Kunc M, Biernat W, Senkus-Konefka E. Estrogen Receptor-Negative Progesterone Receptor-Positive Breast Cancer - "Nobody's Land" or Just an Artifact? Cancer Treat Rev (2018) 67:78-87. doi: 10.1016/j.ctrv.2018.05.005

16. Howlader N, Cronin KA, Kurian AW, Andridge R. Differences in Breast Cancer Survival by Molecular Subtypes in the United States. Cancer Epidemiol Biomarkers Prev (2018) 27:619-26. doi: 10.1158/1055-9965.EPI-17-0627

17. Voduc KD, Cheang MC, Tyldesley S, Gelmon K, Nielsen TO, Kennecke H. Breast Cancer Subtypes and the Risk of Local and Regional Relapse. J Clin Oncol (2010) 28:1684-91. doi: 10.1200/JCO.2009.24.9284

18. Romond EH, Perez EA, Bryant J, Suman VJ, Geyer CE Jr., Davidson NE, et al. Trastuzumab Plus Adjuvant Chemotherapy for Operable HER2-positive Breast Cancer. N Engl J Med (2005) 353:1673-84. doi: 10.1056/NEJMoa052122

19. Swain SM, Baselga J, Kim SB, Ro J, Semiglazov V, Campone M, et al. Pertuzumab, Trastuzumab, and Docetaxel in HER2-positive Metastatic Breast Cancer. N Engl J Med (2015) 372:724-34. doi: 10.1056/NEJMoa1413513

Conflict of Interest: The authors declare that the research was conducted in the absence of any commercial or financial relationships that could be construed as a potential conflict of interest.

Copyright (c) 2021 Zhao and Gong. This is an open-access article distributed under the terms of the Creative Commons Attribution License (CC BY). The use, distribution or reproduction in other forums is permitted, provided the original author(s) and the copyright owner(s) are credited and that the original publication in this journal is cited, in accordance with accepted academic practice. No use, distribution or reproduction is permitted which does not comply with these terms. 